\title{
New analytical technologies for biological discovery
}

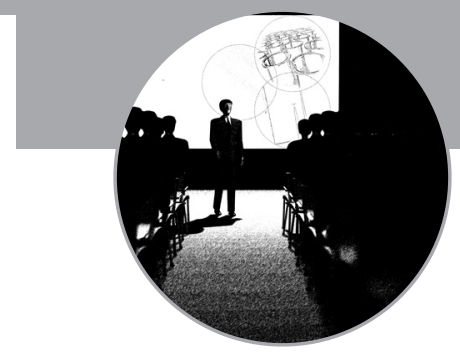

\section{The 16th North American Regional International Society for the Study of Xenobiotics Meeting 2009 18-22 October 2009, Baltimore, Maryland, USA}

While a rapidly growing number of protein and peptide drugs are under development, new analytical technologies have been employed for quantitative measurement of biologics towards understanding of their pharmacokinetics and pharmacodynamics. Besides presenting the recent advance in studies on small molecule xenobiotics, the 16th North American regional meeting of the International Society for the Study of Xenobiotics in Baltimore, Maryland, USA, has provided scientists with the opportunity to share experience in utilization of new technologies for analyses of protein therapeutics and discuss potential application to future biological discovery.

Protein- or peptide-based therapeutics have been recognized as a growing and innovative sector of biotechnology drugs in the pharmaceutical industry. Currently, there are over 130 different proteins or peptides being approved for clinical applications by the US FDA [1]. In addition, a report on 'Medicines in Development Biotechnology', released by the Pharmaceutical Research and Manufacturers of America in 2008 [101], shows that 633 new biological medicines are under clinical investigation, and half of them are proteinbased products (including antibodies). While protein therapeutics exhibit a number of advantages over traditional small-molecule medications (e.g., relatively specific action on the target), understanding the pharmacokinetic and pharmacodynamic properties of protein drugs may encounter various obstacles. One major challenge is that bioanalytical assays for quantification of protein products are often inaccurate, imprecise and nonspecific [2]. Therefore, following the plenary session on an overview of pharmacokinetics and pharmacodynamics of biologics, a particular symposium on new analytical technologies for the next generation of biological discovery was presented at the 16th North American Regional International Society for the Study of Xenobiotics (ISSX) meeting on 19 October 2009 in Baltimore, Maryland, USA [102]. This report is to summarize specific bioanalytical technologies presented at the meeting.

\section{Miniaturized \& automated immunoassay using a Gyros Bioaffy ${ }^{\circledR}$ workstation}

Immunoassay remains the most frequently utilized bioanalytical technique for protein quantification, owing to its ease of performance, high sensitivity and increased specificity, precision and availability [2]. Developed by Gyros AB [103], the Gyros Bioaffy ${ }^{\circledR}$ workstation replaces the 96-well plate used in standard immunoassays with a CD microlaboratory that allows researchers to run protein immunoquantification in nanoliter quantities. The CD microlaboratory that looks like a real CD consists of 96 or 112 individual microstructures. Each microstructure contains a sample inlet, a common channel for liquid distribution, a volume definition chamber, an overflow channel and a column prepacked with streptavidincoated beads. The beads are first activated by biotinylated capture reagents. Analytical samples and fluorophore-labeled detection reagents are then loaded separately. The protein analytes captured by the functionalized column are able to bind to the detection reagents, which are then assayed by a laser-induced fluorescence scanner. The flow-through principle of Gyros Bioaffy workstation helps to reduce the matrix effect and results in an improved signal-to-noise $(\mathrm{S} / \mathrm{N})$ ratio. Lora Hamuro (Merck and Co. Inc.) introduced the miniaturized and automated immunoassay using the Gyros Bioaffy workstation for pharmacokinetic support of protein drugs. They have employed this technology for the analyses of 14 antibody products in four different matrices at Merck. Compared with 96-well enzyme-linked immunosorbant assays, this automated and miniaturized assay using the Gyros Bioaffy workstation not only offers the general benefits of miniaturization and automation (e.g., tenfold lower cost of samples and reagents), less hands-on time and higher efficiency, but also shows good accuracy and precision, a

\author{
Hong-Wu Shen \& \\ Ai-Ming $\mathbf{Y u}^{\dagger}$ \\ ${ }^{\dagger}$ Author for correspondence \\ Department of Pharmaceutical \\ Sciences, School of Pharmacy \\ and Pharmaceutical Sciences, \\ University at Buffalo, The State \\ University of New York, Buffalo, \\ NY, 14260-1200, USA \\ Tel.: + I 7166454817 \\ Fax: + I 7166453693 \\ E-mail: aimingyu@buffalo.edu
}

PHARMACOKINETICS

Dynamic and kinetic mechanisms of exogenous chemical and drug absorption, biological transport, tissue distribution, biotransformation, elimination and toxicology as a function of dosage, and rate of metabolism

Xenobiotics

Chemial substances that are foreign to the biological system. They include naturally occurring compounds, drugs, environmental agents, carcinogens and insecticides, for example

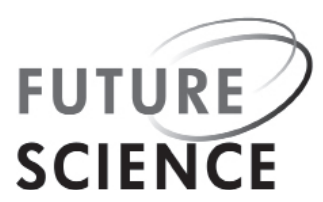




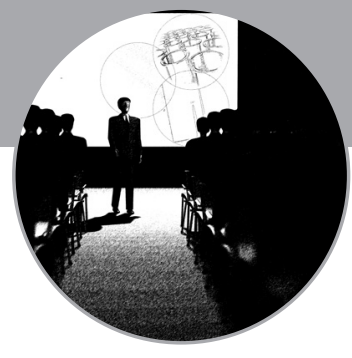

IMMUNOASSAY

Technique using antibodies for identifying or quantifying a substance. Usually the substance being studied serves as an antigen both in antibody production and in measurement of antibody by the test substance broad standard curve, minimal matrix effect, good reproducibility and high throughput. Nevertheless, the major pitfall of immunoassay remains; that is, the potential lack of selectivity toward active, inactive and metabolized proteins, which often depends upon the epitope specificity of the antibody or antibody mimic. Furthermore, the high cost of this technology may limit its broader application in preclinical and clinical testing of protein therapeutics.

\section{LC-MS}

MS-based analytical techniques provide high sensitivity, good selectivity and potential broad application to the analysis of biologics, especially when they are coupled with LC. There are many different types of mass spectrometric instruments on the market. The frequently mentioned mass spectrometers include quadrupole, ion trap (including 3D ion trap, linear ion trap, orbitrap and ion cyclotron resonance ion trap) and TOF mass spectrometers. Richard King (PharmaCadence Analytical Services, LLC) started his presentation by discussing some of the basic characteristics of these instruments and comparing different factors such as mass range, sensitivity, selectivity, linear dynamic range, resolution, mass accuracy and efficiency. The quadrupole mass spectrometers are powerful tools for quantitative analyses because of inherent good selectivity, particularly when tandem mass spectrometers (triple quadrupole) are operated in selected-reaction monitoring (SRM) or multiple-reaction monitoring (MRM) mode. However, quadrupole mass spectrometers exhibit inferior sensitivity when compared with other instruments such as ion trap or TOF mass spectrometers. Sometimes it is difficult to resolve the isotope peaks of a multiple-charged peptide in full-scan mode using a quadrupole mass spectrometer. Furthermore, the MS/MS optimization is still a time-consuming process, despite that MRM or SRM provides good selectivity.

Richard King then talked about the new TOF mass spectrometers that offer acceptable $\mathrm{S} / \mathrm{N}$ ratios based on a combination of high mass resolution and accurate mass information. TOF mass spectrometers also provide comparable quantitative performance as triple quadrupoles when running in selected-ion monitoring mode with a high-resolution setting [3]. With the development of technologies, the limitation of earlier high-resolution MS (HRMS) that needs continuous infusion of a reference mass throughout the entire run was overcome, and new instruments can maintain excellent mass accuracy without recalibration for days. The HRMS not only offers quantitative analysis without extra time spent on MS/MS optimization, but also acquires qualitative information of MS-compatible sample components, such as unknown metabolites. The use of tandem HRMS coupled with a mass defect filter dataprocessing method has successfully identified common and uncommon metabolites from in vitro and in vivo studies $[4,5]$. Besides its application to small molecular compounds, HRMS, such as high-resolution Fourier transform MS, was also applied to the qualitative and quantitative label-free analyses of peptides [6]. However, despite the fast response time, high sensitivity and large mass range, the signal of high-resolution TOF is easily saturated. This drawback leads to a limited dynamic range, and shall be overcome towards qualitative and quantitative measurement of proteins or peptides in different biological systems.

\section{Charge-based detection \& ion-ion reaction as an alternative $M S$ quantitation technique}

Given the fact that the development of a reliable immunoassay is time consuming, traditional immunoassay may not be suitable for early-stage discovery screening of protein or peptide drugs. MS-based assays could provide an alternative approach. However, the SRM method widely used for small molecules may not achieve the sensitivity required for analyses of multiple-charged peptides/proteins because a multiple charging phenomenon disperses the signal intensity and leads to a poor fragmentation efficiency. The multitude of fragments formed from the multiple-charged peptide dilutes the signal and introduces variability. For instance, the multiple-charged precursor ion remains the most abundant ion, and the fragments are very minor ions after MS/MS optimization. Different peptides also have different fragmentation efficiencies. Although protein digestion followed by specific tryptic ion monitoring increases the selectivity and sensitivity, the process is timeconsuming and digestion efficiency also depends on the structure of the protein. Therefore, some novel MS-based techniques have been developed to improve MS detection performance, these were introduced by JC Yves Le Blanc (MDS Analytical Technologies).

A targeted enhanced multiple-charged (targeted EMC) scan using hybrid quadrupole linear ion trap is one of these technologies [7]. 
Besides working as a radio frequency/direct current (RF/DC)-resolving mass filter, the third quadrupole on a quadrupole linear ion trap instrument can also be used to trap the ions by axial ion ejection. After the ions are trapped and thermalized in a linear ion trap, the effective DC trapping barrier depends on their charge states rather than their masses, and adjustment of DC voltage can selectively release the single-charged ions and keep hold of multiple-charged target ions. This process is called EMC scan and is one of the useful functions on the 4000 QTrap $^{\circledR}$. To overcome the limitation of the space-charge effect and reduced ion transmission in conventional EMC scans, the targeted EMC scan modifies this mode by combining the charge separation of EMC with segmented transmission of multiple-charged ions. It fills the ion trap with a selective narrow $\mathrm{m} / \mathrm{z}$ window by optimizing Q1 quadrupole at an open resolution and uses Q3 linear ion trap to achieve charge-based separation. This process is repeated several times in a sequential looped fashion, which generates the complete mass spectrum by Analyst ${ }^{\circledR}$ software. Using a targeted EMC scan, the ion storage and charge separation capabilities are efficiently increased. Signals of multiplecharged tryptic peptides can be efficiently separated from those singly charged chemicals and chemical interferences. Compared with MRM scans, the targeted EMC scan provides desirable MS selectivity for peptides without optimization of collision-induced dissociation for the ion transactions and compoundspecific tuning. Overall, the MS performance of a targeted EMC scan is comparable to the combination of more than two ion transitions in MRM mode.

Another technique relies on ion-ion reactions for charge-state manipulation in the ion trap, as Dr Le Blanc introduced [8]. Through the infusion of a special reagent, the clustering reactions of a multiple-charged peptide/protein can be competed by the oppositely charged ions from the reagent. This ion-ion reaction can be reduced or stopped at a special charge state (ion parking) when the reaction is inhibited by neutralizing the ions and application of an appropriate resonance excitation voltage. The ion parking process accumulates the signal of a specific charge-state ion through reducing the signals of other charge-state ions, which may approach nearly $100 \%$ of the initial multiplecharged reactant abundance. By contrast, the regular process provides product ions that never exceed $60 \%$ of the initial reactant ion abundance. This technique has been utilized for qualitative analyses of peptides and oligonucleotides [9,10], whereas more studies are warranted to employ this technique for quantitative analyses of protein or peptide drugs.

\section{Single-molecule microscopy for the study of antibody trafficking in live cells}

A single-molecule microscopy (SMM) technique was introduced by Sripad Ram (University of Texas Southwestern Medical Center) as a useful tool to capture the intracellular behavior of bio-molecules in live cells. Combining the advances in imaging technology, computer control of complex experiments and fluorescent labeling methodology, SMM is widely used to study the localization and behavior of a single molecule in a cellular environment. Using this technique, they have successfully investigated the intracellular localization of $\operatorname{IgG}$ and neonatal $F_{c}$ receptor $(F c R n)$ in a human endothelial cell line HMEC-1 [11]. Note that FcRn receptor is known to play a critical role in transport of IgGs across and within cells. When SMM was used along with total internal reflection fluorescence microscopy [12], the dynamics of IgG exocytosis were obtained to assess the molecular and cellular mechanisms involved in the process. Their data showed that IgG exocytosed through rapid fusion of FcRn-containing vesicles or tubules with the plasma membrane (full-fusion mode) and/or slow release with partial mixing of membrane contents (prolonged-release mode). The newly developed multifocal plane microscopy technique has been used to simultaneously image different plasma membrane planes within cells, toward monitoring and characterization of intracellular events involving IgG and FcRn interaction [13]. These new imaging tools hold great promise for visualization and assessment of dynamics of proteins and peptides in different pathways within live cells.

\section{Summary}

With the growing interest in discovery and development of protein and peptide drugs, new analytical technologies have been used for qualitative and quantitative analyses of these biologics towards understanding of their pharmacokinetics and pharmacodynamics. One is to take advantage of those emerging MS technologies to develop MS-based assays with increased sensitivity and selectivity, and 
employ these assays for daily analyses of protein therapeutics. Meanwhile, miniaturization and automation of traditional immunoassays has facilitated the analysis of protein and peptide drugs. In addition, single-molecule imaging technologies may eventually be applied to understanding the processing of endobiotic and xenobiotic proteins in live cells for the next generation of biological discovery. Nevertheless, these methods might not be able to distinguish the active proteins from inactive or metabolized forms. Complementary biological activity assays could help evaluate the levels of active proteins and improve the understanding of protein pharmacokinetics and pharmacodynamics [2].

\section{Financial \& competing interests disclosure}

The authors have no relevant affliations or financial involvement with any organization or entity with a financial interest in or financial conflict with the subject matter or materials discussed in the manuscript. This includes employment, consultancies, honoraria, stock ownership or options, expert testimony, grants or patents received or pending, or royalties.

No writing assistance was utilized in the production of this manuscript.

\section{Bibliography}

1 Leader B, Baca QJ, Golan DE. Protein therapeutics: a summary and pharmacological classification. Nat. Rev. Drug Discov. 7(1), 21-39 (2008).

2 Tang L, Persky AM, Hochhaus G, Meibohm B. Pharmacokinetic aspects of biotechnology products. J. Pharm. Sci. 93(9), 2184-2204 (2004).

3 Zhang N, Fountain ST, Bi H, Rossi DT. Quantification and rapid metabolite identification in drug discovery using API time-of-flight LC/MS. Anal. Chem. 72(4), 800-806 (2000).

4 Zhu M, Ma L, Zhang D et al. Detection and characterization of metabolites in biological matrices using mass defect filtering of liquid chromatography/high resolution mass spectrometry data. Drug Metab. Dispos. 34(10), 1722-1733 (2006).

5 Bateman KP, Castro-Perez J, Wrona M et al. MSE with mass defect filtering for in vitro and in vivo metabolite identification. Rapid Commun. Mass Spectrom. 21(9), 1485-1496 (2007).
6 Meng F, Wiener MC, Sachs JR et al. Quantitative analysis of complex peptide mixtures using FTMS and differential mass spectrometry. J. Am. Soc. Mass Spectrom. 18(2), 226-233 (2007).

7 Drogaris P, Le Blanc JC, Fitzgerald JE et al. Enhanced protein detection using a trapping mode on a hybrid quadrupole linear ion trap (Q-trap). Anal. Chem. 81(15), 6300-6309 (2009)

8 Mcluckey SA, Reid GE, Wells JM. Ion parking during ion/ion reactions in electrodynamic ion traps. Anal. Chem. 74(2), 336-346 (2002).

9 Mcluckey SA, Wu J, Bundy JL, Stephenson JL Jr, Hurst GB. Oligonucleotide mixture analysis via electrospray and ion/ion reactions in a quadrupole ion trap. Anal. Chem. 74(5), 976-984 (2002).

10 Xia Y, Chrisman PA, Erickson DE et al. Implementation of ion/ion reactions in a quadrupole/time-of-flight tandem mass spectrometer. Anal. Chem. 78(12), 4146-4154 (2006).
11 Ober Rj, Martinez C, Vaccaro C, Zhou J, Ward ES. Visualizing the site and dynamics of IgG salvage by the MHC class i-related receptor, fcrn. J. Immunol. 172(4), 2021-2029 (2004).

12 Ober RJ, Martinez C, Lai X, Zhou J, Ward ES. Exocytosis of IgG as mediated by the receptor, FcRn: An analysis at the single-molecule level. Proc. Natl Acad. Sci. USA 101(30), 1107611081 (2004).

13 Prabhat P, Gan Z, Chao J et al. Elucidation of intracellular recycling pathways leading to exocytosis of the $\mathrm{Fc}$ receptor, FcRn, by using multifocal plane microscopy. Proc. Natl Acad. Sci. USA 104(14), 5889-5894 (2007).

\section{- Websites}

101 Tauzin B. Biotechnology research continues to bolster arsenal against disease with 633 medicines in development (2008) www.phrma.org/files/attachments/ Biotech\%202008.pdf

102 16th North American Regional ISSX Meeting www.issx.org

103 Gyros www.gyros.com/en/home/index.html 